\title{
Termodinâmica Estendida de Fluidos Viscosos com Condução de Calor
}

A. VIGNATTI 1 , Departamento de Matemática da UFES, 29060-900 Vitória, ES, Brasil.

I-SHIH LIU², Instituto de Matemática da UFRJ, 21945-970 Rio de Janeiro, RJ, Brasil.

Resumo. Termodinâmica estendida é uma teoria fenomenológica com o objetivo principal de determinar os campos de deformação, temperatura, tensão e fluxo de calor. As equações da termodinâmica estendida consistem das leis usuais de conservação de massa, momento e energia e um conjunto de equações de balanço adicionais, e tem sido aplicadas para a formulação de termodinâmica estendida de fluidos $[1],[6]$ bem como sólidos viscoelásticos. Aqui será formulada uma teoria similar para fluido viscoso com condução de calor. Propagação de ondas em gases ideais tem sido analisada.

\section{Introdução}

A termodinâmica estendida para gases ideais monatômicos, como uma teoria de 13 momentos, i.e., densidade, velocidade, tensor tensão e fluxo de calor, foi primeiramente formulada por Liu e Müller [5]. A teoria proposta neste artigo trata também os fluidos viscosos. Porém, em vez de identificar a energia com o traço do momento de segunda ordem (tensor tensão), a energia é considerada como um campo independente. Assim, além de 13 equações de balanço usuais, é aumentada a equação da energia total, e conseqüentemente, a teoria consiste num sistema de 14 equações de balanço.

Não muito diferente da teoria proposta em [1], a análise do presente trabalho baseia-se no procedimento proposto em [3], o que facilita a exploração do princípio de entropia para deduzir as equações constitutivas.

Aqui é usada a notação usual da soma sobre índices repetidos. Parênteses indicam a simetrização $A_{(i j)}=\frac{1}{2}\left(A_{i j}+A_{j i}\right)$ e o símbolo \langle\rangle indica a simetrização sem traço $A_{\langle i j\rangle}=A_{(i j)}-\frac{1}{3} A_{s s} \delta_{i j}$.

\footnotetext{
1aldo@cce.ufes.br

${ }^{2}$ liu@im.ufrj.br
} 


\section{Equações de Balanço}

As leis de conservação de massa, momento linear e energia em um sistema de coordenadas espaciais $\left(x_{i}, t\right)$ relativo a um referencial inercial e livres de suprimento externo, são escritas como

$$
\begin{gathered}
\frac{\partial \varrho}{\partial t}+\frac{\partial \varrho v_{k}}{\partial x_{k}}=0 \\
\frac{\partial \varrho v_{i}}{\partial t}+\frac{\partial}{\partial x_{k}}\left(\varrho v_{i} v_{k}-T_{i k}\right)=0 \\
\frac{\partial \varrho e}{\partial t}+\frac{\partial}{\partial x_{k}}\left(\varrho e v_{k}-v_{i} T_{i k}+q_{k}\right)=0
\end{gathered}
$$

onde $e=\left(v^{2} / 2+\varepsilon\right)$ é a energia total específica.

Nós consideramos um estado de um fluido viscoso condutor de calor caracterizado pelos 14 campos $\left(\varrho, v_{i}, \theta, T_{i j}, q_{j}\right)$, que representam a densidade de massa, a velocidade, a temperatura, o tensor tensão de Cauchy e o fluxo de calor, respectivamente. Estas equações estão baseadas nas leis de conservação (2.1) e nas equações

$$
\begin{gathered}
\frac{\partial F_{i j}}{\partial t}+\frac{\partial}{\partial x_{k}}\left(F_{i j} v_{k}+G_{i j k}\right)=P_{i j}, \\
\frac{\partial F_{i i j}}{\partial t}+\frac{\partial}{\partial x_{k}}\left(F_{i i j} v_{k}+G_{i i j k}\right)=P_{i i j},
\end{gathered}
$$

para os momentos $F_{i j}, F_{i i j}$, seus fluxos $G_{i j k}, G_{i i j k}$ e produções $P_{i j}$ e $P_{i i j}$, que são acrescentadas para construirmos uma teoria estendida de 14 momentos para fluidos viscosos com condução de calor.

O requerimento da invariância Galileana do sistema de equações de balanço (2.1)-(2.2) implica a seguinte dependência explícita da velocidade $v_{i}$ (ver [6]),

$$
\begin{aligned}
F_{i j} & =\varrho v_{i} v_{j}+\varrho_{i j}, \\
G_{i j k} & =v_{i} p_{j k}+v_{j} p_{i k}+p_{i j k}, \\
P_{i j} & =\pi_{i j}, \\
F_{i i j} & =\varrho v_{i} v_{i} v_{j}+2 \varrho_{i j} v_{i}+\varrho_{i i} v_{j}+\varrho_{i i j}, \\
G_{i i j k} & =v_{i} v_{i} p_{j k}+2 v_{i} v_{j} p_{i k}+2 v_{i} p_{i j k}+v_{j} p_{i i k}+p_{i i j k}, \\
P_{i i j} & =\pi_{i i j}+3 v_{(i} \pi_{i j)},
\end{aligned}
$$

onde, respectivamente, $\varrho_{i j}, \varrho_{i i j}, p_{i j k}, p_{i i j k}, \pi_{i j}, \pi_{i i j}$ são as partes internas dos momentos $F_{i j}$ e $F_{i i j}$, dos fluxos $G_{i j k}$ e $G_{i i j k}$ e das produções $P_{i j}$ e $P_{i i j}$. Motivados pela teoria cinética de gases, admitiremos que $F_{i j}, G_{i j k}, P_{i j}, F_{i i j}, G_{i i j k}, P_{i i j}$ são simétricos nos índices $i, j$. Admitiremos ainda que as quantidades $\varrho_{i j}, p_{i j}=-T_{i j}$, $p_{i j k}, \pi_{i j}, p_{i i k}, p_{i i j k}, \pi_{i i j}$ assim como $\varrho, \varepsilon, T_{i k}, q_{k}$ são quantidades objetivas.

Em vez de considerar $\left(\varrho, v_{i}, \theta, T_{i j}, q_{j}\right)$ como as variáveis campos básicos do sistema (2.1)-(2.2), nós podemos escolher as variáveis $\left(\varrho, v_{i}, \theta, F_{i j}, F_{i i j}\right)$, por conveniência, assumindo uma certa invertibilidade usual das funções constitutivas. Portanto o sistema (2.1)-(2.2) será completado pelas relações constitutivas

$$
\mathcal{C}=\tilde{\mathcal{C}}\left(\varrho, v_{i}, \theta, F_{i j}, F_{i i j}\right)
$$


Aqui $\mathcal{C}$ representa as quantidades constitutivas $\left\{\varrho_{i j}, q_{k}, T_{i j}, p_{i j k}, \pi_{i j}, G_{i i j k}, \pi_{i i j}\right\}$.

Todo campo $\left(\varrho, v_{i}, \theta, F_{i j}, F_{i i j}\right)$ que satisfaça o sistema (2.1)-(2.2) junto com as relações constitutivas (2.3), será chamado processo termodinâmico.

\section{Princípio de Entropia}

O princípio de entropia estabelece que para todo processo termodinâmico a inequação de entropia

$$
\frac{\partial \varrho \eta}{\partial t}+\frac{\partial}{\partial x_{k}}\left(\varrho \eta v_{k}+\Phi_{k}\right)=\Sigma \geq 0
$$

deve ser válida, onde o fluxo de entropia $\Phi_{\kappa}$, a produção de entropia $\Sigma$ e a densidade de entropia específica $\eta$, são também dadas pelas relações constitutivas da forma (2.3). Além disso, a função $h=\varrho \eta$ é admitida ser côncava nas variáveis campos básicos e tanto $h$ quanto $\Phi=\left(\Phi_{1}, \Phi_{2}, \Phi_{3}\right)$ são admitidas ser objetivas.

As equações (2.1) e (2.2) podem ser escritas na forma compacta,

$$
\frac{\partial \boldsymbol{u}}{\partial t}+\frac{\partial}{\partial x_{k}}\left(\boldsymbol{u} v_{k}+\boldsymbol{G}_{k}\right)=\boldsymbol{P},
$$

com $\boldsymbol{u}=\left(\varrho, \varrho v_{i}, \varrho e, F_{i j}, F_{i i j}\right), \boldsymbol{G}_{k}=\left(0,-T_{i k},-v_{i} T_{i k}+q_{k}, G_{i j k}, G_{i i j k}\right)$, e $\boldsymbol{P}=$ $\left(0,0,0, P_{i j}, P_{i i j}\right)$. O princípio de entropia implica na existência de um multiplicador de Lagrange [2],[3] $\boldsymbol{\Lambda}$ tal que

$$
d h=\boldsymbol{\Lambda} \cdot d \boldsymbol{u}, \quad d \Pi_{k}=\boldsymbol{\Lambda} \cdot d \boldsymbol{H}_{k} \quad \text { e } \quad \Sigma=\boldsymbol{\Lambda} \cdot \boldsymbol{P},
$$

onde, $\Pi_{k}=h v_{k}+\Phi_{k}$ e $\boldsymbol{H}_{k}=\boldsymbol{u} v_{k}+\boldsymbol{G}_{k}$.

Cada uma das quantidades $\varrho, \varepsilon, \varrho_{i j}, \varrho_{i i j}, h$ e $\Phi_{k}$ é objetiva e em particular independente da velocidade, o que implica na existência de multiplicadores de Lagrange $\lambda_{\varrho}, \Lambda, \Lambda_{i j}, \lambda_{j}, \Lambda_{i}$ tais que

$$
\begin{aligned}
d h= & \lambda_{\varrho} d \varrho+\Lambda d(\varrho \varepsilon)+\Lambda_{i j} d \varrho_{i j}+\lambda_{j} d \varrho_{i i j} \\
0= & \varrho \Lambda_{i}+3 \lambda_{(i} \varrho_{j j)} \\
d \Phi_{k}= & \Lambda d q_{k}+\Lambda_{i} d p_{i k}+\Lambda_{i j} d p_{i j k}+\lambda_{j} d p_{i i j k} \\
0= & \left(\lambda_{\varrho} \varrho+\Lambda \varrho \varepsilon+\Lambda_{s j} \varrho_{s j}+\lambda_{j} \varrho_{s s j}-h\right) \delta_{i k} \\
& +\Lambda p_{i k}+2 \Lambda_{i j} p_{j k}+2 \lambda_{j} p_{i j k}+\lambda_{i} p_{j j k} \\
\Sigma= & \Lambda_{i j} \pi_{i j}+\lambda_{j} \pi_{i i j} \geq 0 .
\end{aligned}
$$

O equilíbrio é definido como um processo termodinâmico sem produção de entropia. Da relação $(3.2)_{5}$, tem-se que a produção de entropia atinge seu valor mínimo no equilíbrio, isto é, quando $\pi_{i j}=0$ e $\pi_{i i j}=0$ para todo $i, j=1,2,3$.

Assim como $\Sigma$, as funções $\pi_{i j}, \pi_{i i j}$, a priori, dependem das variáveis $\lambda_{\varrho}, \Lambda, \Lambda_{i j}$, $\lambda_{j}$ e $\Lambda_{i}$. Será assumido que $\pi_{i j}$ é invertível na variável $\Lambda_{i j}$ e que $\pi_{i i j}$ é invertível na variável $\lambda_{j}$ para trocar $\Lambda_{i j}, \lambda_{j}$ por $\pi_{i j}, \pi_{i i j}$, respectivamente, como variáveis em $\Sigma$. Então a condição necessária para o mínimo da produção de entropia implica que

$$
0=\left.\Lambda_{i j}\right|_{E}, \quad 0=\left.\lambda_{j}\right|_{E} \quad \text { e }\left.\quad \Lambda_{k}\right|_{E}=0 .
$$


As relações $(3.2)_{1,4}$ avaliadas no equilíbrio reduzem-se para

$$
\left.d h\right|_{E}=\left.\lambda_{\varrho}\right|_{E} d \varrho+\left.\Lambda\right|_{E} d(\varrho \varepsilon) \quad \text { e }\left.\quad p_{i k}\right|_{E}=p_{0} \delta_{i k} .
$$

A relação de Gibbs

$$
d h_{0}=\frac{1}{\theta}(d(\varrho \varepsilon)-g d \varrho)
$$

onde $h_{0}=\left.h\right|_{E}$, é decorrente da teoria ordinária [3], em que $g=\psi+\frac{p_{0}}{\varrho}$ é a entalpia livre, $\psi=\varepsilon-\theta \eta_{0}$ é a energia livre, $\eta_{0}=\left.\eta\right|_{E}$ é uma função dependente somente de $\varrho$ e $\theta$ assim como $h_{0}$, $\varepsilon$ e $p_{0}$. Comparamos $(3.4)_{1}$ com a relação de Gibbs e fizemos as identificações

$$
\left.\Lambda\right|_{E}=\frac{1}{\theta} \quad \text { e }\left.\quad \lambda_{\varrho}\right|_{E}=-\frac{1}{\theta} g
$$

Será formulada aqui a teoria para processo termodinâmico "perto" do equilíbrio. Matematicamente, isto significa que as variáveis não-equilíbrio, ou seja, as variáveis que se anulam quando avaliadas no equilíbrio, serão consideradas como quantidades pequenas de primeira ordem e, funções constitutivas serão representadas em termos delas até certa ordem superior. Os multiplicadores de Lagrange $\left(\Lambda_{i j}, \lambda_{j}, \Lambda_{j}\right)$ são quantidades não-equilíbrio.

São definidas as quantidades não-equilíbrio $\Lambda_{\varepsilon}, \Lambda_{\varrho}, S_{i j}, \varrho_{\varepsilon}$ de modo que

$$
\Lambda=\Lambda_{\varepsilon}+\frac{1}{\theta}, \quad \lambda_{\varrho}=\Lambda_{\varrho}-\frac{1}{\theta} g, \quad p_{i j}=p_{0} \delta_{i j}-S_{i j}, \quad \varrho_{s s}=\varrho_{\varepsilon}-\left.\varrho_{s s}\right|_{E} .
$$

A objetividade de $\varrho_{i j}$ e $\varrho_{i i j}$ implica que $\left.\varrho_{\langle i j\rangle}\right|_{E}$ e $\left.\varrho_{i i j}\right|_{E}=0$.

A seguir serão representadas, em termos das quantidades não-equilíbrio $\varrho_{\varepsilon}, \varrho_{\langle i j\rangle}$, $\varrho_{i i j}, \Lambda_{\varepsilon}, \Lambda_{i}, \Lambda_{\langle i j\rangle}, \Lambda_{j j}$ e $\lambda_{j}$, as funções densidade de entropia $h$ e a conjugada do fluxo de entropia $\Phi_{k}$.

A relação $(3.2)_{1}$ torna-se

$$
\begin{aligned}
d h= & \left(\Lambda_{\varrho}+\theta \Lambda_{\varepsilon} \frac{\partial h_{0}}{\partial \varrho}+\frac{\partial h_{0}}{\partial \varrho}+\frac{1}{3} \Lambda_{i i} \frac{\left.\partial \varrho_{s s}\right|_{E}}{\partial \varrho}+\Lambda_{\varepsilon} g\right) d \varrho \\
& +\left(\theta \Lambda_{\varepsilon} \frac{\partial h_{0}}{\partial \theta}+\frac{\partial h_{0}}{\partial \theta}+\frac{1}{3} \Lambda_{i i} \frac{\partial \varrho_{s s} \mid E}{\partial \theta}\right) d \theta+\Lambda_{\langle i j\rangle} d \varrho_{\langle i j\rangle}+\frac{1}{3} \Lambda_{i i} d \varrho_{\varepsilon}+\lambda_{j} d \varrho_{i i j}
\end{aligned}
$$

Definimos a conjugada do fluxo de entropia $\Phi_{k}$ por

$$
\hat{\Phi}_{k}=\Lambda q_{k}+\Lambda_{i} p_{i k}+\Lambda_{i j} p_{i j k}+\lambda_{j} p_{i i j k}-\Phi_{k}
$$

e usamos as equações $(3.2)_{3},(3.5)_{1}$, para termos que

$$
d \hat{\Phi}_{k}=-\frac{1}{\theta^{2}} q_{k} d \theta+q_{k} d \Lambda_{\varepsilon}+p_{i k} d \Lambda_{i}+p_{\langle i j\rangle k} d \Lambda_{\langle i j\rangle}+\frac{1}{3} p_{i i k} d \Lambda_{j j}+p_{i i j k} d \lambda_{j}
$$

ou seja, que $\hat{\Phi}_{k}=\hat{\Phi}_{k}\left(\varrho, \theta, \Lambda_{\varepsilon}, \Lambda_{i}, \Lambda_{\langle i j\rangle}, \Lambda_{j j}, \lambda_{j}\right)$, com $\hat{\Phi}_{k}$ independente de $\varrho$. 


\section{Equações Constitutivas}

Para as equações constitutivas lineares, serão necessárias e suficientes as representações de $h$ e $\hat{\Phi}_{k}$ mediante termos até segunda ordem.

Assumimos que $\varrho_{\varepsilon}, \varrho_{\langle i j\rangle}, \varrho_{i i j}, \Lambda_{\varepsilon}, \Lambda_{i}, \Lambda_{\langle i j\rangle}, \Lambda_{j j}$ e $\lambda_{j}$ são quantidades pequenas de mesma ordem, o que facilita escrevermos as representações das funções isotrópicas $h$ e $\hat{\Phi}=\left(\hat{\Phi}_{1}, \hat{\Phi}_{2}, \hat{\Phi}_{3}\right)$ até segunda ordem (veja [3]),

$$
\begin{aligned}
h= & h_{0}+k_{0} \varrho_{\varepsilon}+h_{1} \varrho_{i i j} \varrho_{n n j}+h_{2} \varrho_{\langle i j\rangle} \varrho_{\langle i j\rangle}+h_{3} \varrho_{\varepsilon}^{2}+o(3), \\
\hat{\Phi}_{k}= & \alpha \lambda_{k}+\beta \Lambda_{k}+a_{1} \Lambda_{\varepsilon} \lambda_{k}+b_{1} \Lambda_{\varepsilon} \Lambda_{k}+a_{2} \Lambda_{\langle k i\rangle} \lambda_{i}+b_{2} \Lambda_{\langle k i\rangle} \Lambda_{i} \\
& +a_{3} \Lambda_{i i} \lambda_{k}+b_{3} \Lambda_{i i} \Lambda_{k}+o(3),
\end{aligned}
$$

onde os coeficientes $k_{0}, h_{n}, \alpha, \beta, a_{n}, b_{n}$, são funções de $(\varrho, \theta)$. A notação $o(n)$ representa termos de ordem maior ou igual a $n$ nas quantidades $\varrho_{\varepsilon}, \varrho_{\langle i j\rangle}, \varrho_{i i j}, \Lambda_{\varepsilon}, \Lambda_{i}$, $\Lambda_{\langle i j\rangle}, \Lambda_{j j}$ e $\lambda_{j}$.

Comparamos (3.6) com (4.1) $)_{1}$ e obtivemos as expressões

$$
\begin{aligned}
\lambda_{j} & =2 h_{1} \varrho_{i i j}+o(2) \\
\Lambda_{i i} & =3 k_{0}+6 h_{3} \varrho_{\varepsilon}+o(2) \\
\Lambda_{\langle i j\rangle} & =2 h_{2} \varrho_{\langle i j\rangle}+o(2), \\
\Lambda_{\varepsilon} & =-2 c_{\theta} h_{3} \varrho_{\varepsilon}+o(2), \\
\Lambda_{\varrho} & =\left[\left(\theta \frac{\partial h_{0}}{\partial \varrho}+g\right) c_{\theta}-\frac{\partial \varrho_{s s} \mid}{\partial \varrho}\right] 2 h_{3} \varrho_{\varepsilon}+o(2),
\end{aligned}
$$

para os multiplicadores de Lagrange, onde $c_{\theta}=\left(\frac{\partial h_{0}}{\partial \theta}\right)^{-1} \frac{1}{\theta} \frac{\left.\partial \varrho_{s s}\right|_{E}}{\partial \theta}$. Avaliamos $(4.2)_{2}$ no equilíbrio e vimos que $k_{0}=0$. Usamos $(3.2)_{2}$, para encontrarmos que

$$
\Lambda_{i}=-\frac{10}{3} \frac{\left.\varrho_{s s}\right|_{E}}{\varrho} h_{1} \varrho_{j j i}+o(2) .
$$

Comparamos (3.7) com $(4.1)_{2}$ e obtivemos as expressões

$$
\begin{aligned}
q_{k} & =-\theta^{2}\left(\frac{\partial \alpha}{\partial \theta} \lambda_{k}+\frac{\partial \beta}{\partial \theta} \Lambda_{k}\right)+o(2), \\
q_{k} & =a_{1} \lambda_{k}+b_{1} \Lambda_{k}+o(2), \\
p_{i k} & =\beta \delta_{i k}+b_{1} \Lambda_{\varepsilon} \delta_{i k}+b_{2} \Lambda_{\langle k i\rangle}+b_{3} \Lambda_{j j} \delta_{i k}+o(2), \\
p_{\langle i j\rangle k} & =a_{2} \delta_{k\langle i} \lambda_{j\rangle}+b_{2} \delta_{k\langle i} \Lambda_{j\rangle}+o(2), \\
p_{i i k} & =3 a_{3} \lambda_{k}+3 b_{3} \Lambda_{k}+o(2), \\
p_{i i j k} & =\left(\alpha+a_{1} \Lambda_{\varepsilon}\right) \delta_{k j}+a_{2} \Lambda_{\langle k j\rangle}+a_{3} \Lambda_{i i} \delta_{k j}+o(2), \\
0 & =\frac{\partial \hat{\Phi}_{k}}{\partial \varrho}=\frac{\partial \alpha}{\partial \varrho} \lambda_{k}+\frac{\partial \beta}{\partial \varrho} \Lambda_{k}+o(2),
\end{aligned}
$$

para os fluxos. Logo por $(3.4)_{2}, \beta=p_{0}$.

As equações $(4.3)_{1,2,7}$ implicam que

$$
a_{1}-\frac{5}{3} \frac{\left.\varrho_{s s}\right|_{E}}{\varrho} b_{1}=-\theta^{2}\left(\frac{\partial \alpha}{\partial \theta}-\frac{5}{3} \frac{\left.\varrho_{s s}\right|_{E}}{\varrho} \frac{\partial p_{0}}{\partial \theta}\right), \quad 0=\frac{\partial \alpha}{\partial \varrho}-\frac{5}{3} \frac{\left.\varrho_{s s}\right|_{E}}{\varrho} \frac{\partial p_{0}}{\partial \varrho} .
$$


A parte linear da simetrização sem traço, e do traço de $(3.2)_{4}$, reduzem-se respectivamente a

$$
\begin{aligned}
b_{2} & =-2 p_{0} \theta, \\
\varrho \frac{\left.\partial \varrho_{s s}\right|_{E}}{\partial \varrho}-\left.\varrho_{s s}\right|_{E}+\left(c_{\theta}-2\right) \beta & =\frac{1}{\theta}\left(3 b_{3}-c_{\theta} b_{1}\right)+\varrho^{2} \frac{\partial \varepsilon}{\partial \varrho} c_{\theta} .
\end{aligned}
$$

A partir do tensor viscoso $S_{i j}$, definido em $(3.5)_{3}$, é definida a pressão dinâmica $p_{d}=-\frac{1}{3} S_{k k}$. Pelas relações $(3.5)_{3},(4.3)_{3}$ e $(4.2)_{2,3,4}$, tem-se

$$
p_{i j}=\left(p_{0}+p_{d}\right) \delta_{i j}-S_{\langle i j\rangle} \quad \text { e } \quad p_{d}=\left(3 b_{3}-c_{\theta} b_{1}\right) 2 h_{3} \varrho_{\varepsilon}+o(2) .
$$

A equação $(4.3)_{7}$ escrita mediante termos de segunda ordem, implica que

$$
\begin{gathered}
h_{2}\left(\frac{\partial a_{2}}{\partial \varrho}-\frac{5}{3} \frac{\left.\varrho_{s s}\right|_{E}}{\varrho} \frac{\partial b_{2}}{\partial \varrho}\right)-\frac{1}{\varrho} \frac{\partial \beta}{\partial \varrho}=0 \\
\frac{5}{3} \frac{1}{\varrho} \frac{\partial \beta}{\partial \varrho}+2 c_{\theta} h_{3}\left(\frac{\partial a_{1}}{\partial \varrho}-\frac{5}{3} \frac{\left.\varrho_{s s}\right|_{E}}{\varrho} \frac{\partial b_{1}}{\partial \varrho}\right)-6 h_{3}\left(\frac{\partial a_{3}}{\partial \varrho}-\frac{5}{3} \frac{\left.\varrho_{s s}\right|_{E}}{\varrho} \frac{\partial b_{3}}{\partial \varrho}\right)=0 .
\end{gathered}
$$

Definindo $A_{i}=a_{i}-\frac{5}{3} \frac{\left.\varrho_{s s}\right|_{E}}{\varrho} b_{i}$, as equações (4.3) tornam-se as seguintes equações constitutivas lineares em termos dos campos básicos $\left(\varrho, \theta, q_{k}, S_{\langle i k\rangle}, p_{d}\right)$ :

$$
\begin{array}{rlrl}
\varrho_{i i k} & =\frac{1}{2 A_{1} h_{1}} q_{k}, & p_{i j k} & =\frac{A_{2}}{A_{1}} q_{\langle i} \delta_{j\rangle k}+\frac{A_{3}}{A_{1}} q_{k} \delta_{i j}, \\
\varrho_{\langle i k\rangle}=\frac{1}{4 p_{0} \theta h_{2}} S_{\langle i k\rangle}, & p_{i i j k}=\left(\alpha+\frac{3 a_{3}-c_{\theta} a_{1}}{3 b_{3}-c_{\theta} b_{1}} p_{d}\right) \delta_{j k}+\frac{a_{2}}{2 p_{0} \theta} S_{\langle j k\rangle} .
\end{array}
$$

\section{Concavidade da Densidade de Entropia}

Além da condição (3.3), para que $\Sigma$ seja mínima no equilíbrio, é exigido também que $\left.\frac{\partial^{2} \Sigma}{\partial X_{A} \partial X_{B}}\right|_{E} \geq 0$, onde $X_{A}$ é da forma $\left(\Lambda_{\varrho}, \Lambda_{\varepsilon}, \Lambda_{i j}, \lambda_{j}\right)$.

As funções $\pi_{i j}$ e $\pi_{i i j}$ dependem de $\left(\Lambda_{\varrho}, \Lambda_{\varepsilon}, \Lambda_{i j}, \lambda_{j}\right)$. Então as representações lineares de $\pi_{i j}$ e $\pi_{i i j}$ são dadas por

$$
\pi_{i j}=r_{1} \Lambda_{i j}+o(2), \quad \pi_{i i j}=\tau \lambda_{j}+o(2),
$$

onde $r_{1}$ e $\tau$ são funções de $(\varrho, \theta)$ com

$$
r_{1}>0 \quad \text { e } \quad \tau>0
$$

A hipótese, $h(\boldsymbol{u})$ ser côncava na variável $\boldsymbol{u}$, admitida na Seção 3 , equivale a afirmar que o sistema (3.1) é hiperbólico simétrico, ou ainda que $\delta \boldsymbol{\Lambda} \cdot \delta \boldsymbol{u}<0$ para todas as variações $\delta \boldsymbol{\Lambda}, \delta \boldsymbol{u}$. É desenvolvido o produto interno $\delta \boldsymbol{\Lambda} \cdot \delta \boldsymbol{u}$ usando que $\delta \boldsymbol{\Lambda}=\left(\delta \hat{\lambda}_{\varrho}, \delta \hat{\Lambda}_{i}, \delta \hat{\Lambda}, \delta \hat{\Lambda}_{i j}, \delta \hat{\lambda}_{j}\right)$ e $\delta \boldsymbol{u}=\left(\delta \varrho, \delta\left(\varrho v_{i}\right), \delta(\varrho e), \delta F_{i j}, \delta F_{i i j}\right)$, e obtido

$$
h_{1}<0, \quad h_{2}<0, \quad h_{3}<0, \quad \frac{\partial g}{\partial \varrho}>0, \quad \frac{\partial \varepsilon}{\partial \theta}>0 .
$$


A relação $(5.2)_{5}$ implica $\frac{\partial h_{0}}{\partial \theta}>0$, além de mostrar que o calor específico $c_{v}=\frac{\partial \varepsilon}{\partial \theta}$ a volume constante, é positivo. As definições de $g, \psi$ e $\eta_{0}$ implicam $\frac{\partial p_{0}}{\partial \varrho}=\varrho \frac{\partial g}{\partial \varrho}>0$, ou seja, que a compressibilidade isotérmica também é positiva.

\section{Equações Diferenciais dos Campos}

Substituindo as equações constitutivas (4.4) nas equações de balanço (2.2), será obtido um sistema de equações diferenciais parciais quase-linear para os campos $\varrho$, $\theta, v_{i}, p_{d}, S_{\langle i j\rangle}, q_{j}$. O campo de equações será linearizado ao excluir todos os termos não lineares, tais como produtos de $p_{d}, q_{j}, S_{\langle i j\rangle}$ com as derivadas de $\varrho, \theta$ e $v_{i}$. Isto fornecerá as últimas equações para um fluido viscoso condutor de calor. A saber,

$$
\begin{aligned}
-S_{\langle i j\rangle} & =\tau_{s} \dot{S}_{\langle i j\rangle}-2 \mu \frac{\partial v_{\langle i}}{\partial x_{j\rangle}}-2 \mu K \frac{\partial q_{\langle i}}{\partial x_{j\rangle}}, \\
-p_{d} & =\tau_{\pi} \dot{p}_{d}+\nu \frac{\partial v_{k}}{\partial x_{k}}-N L \frac{\partial q_{k}}{\partial x_{k}}, \\
-q_{j} & =\tau_{q} \dot{q}_{j}+\kappa \frac{\partial \theta}{\partial x_{j}}-\kappa L \theta \frac{\partial p_{d}}{\partial x_{j}}-\kappa K \theta \frac{\partial S_{\langle j k\rangle}}{\partial x_{k}},
\end{aligned}
$$

onde foram introduzidas as seguintes notações para os coeficientes:

$$
\tau_{s}=-\frac{1}{2 h_{2} r_{1}}, \quad \tau_{\pi}=-\frac{1}{6 r_{1} h_{3}}, \quad \tau_{q}=-\frac{1}{2 h_{1} \tau},
$$

para os tempos de relaxamento do tensor cizalhante, do fluxo de calor e da pressão dinâmica, e

$$
\mu=\frac{2 p_{0}^{2} \theta}{r_{1}}, \quad \kappa=\frac{A_{1}^{2}}{\theta^{2} \tau}, \quad \nu=\frac{1}{3 \theta r_{1}}\left(3 b_{3}-c_{\theta} b_{1}\right)^{2},
$$

para a viscosidade cizalhante, a condutividade térmica e a viscosidade volumétrica respectivamente, e três funções térmico-viscosa,

$$
K=\frac{A_{2}}{2 p_{0} A_{1}}, \quad L=\frac{\theta}{A_{1}} \frac{3 A_{3}-c_{\theta} A_{1}}{3 b_{3}-c_{\theta} b_{1}}, \quad N=\nu .
$$

A partir das desigualdades (5.1), (5.2) $)_{1,2,3}$ tem-se: $\tau_{s}>0, \tau_{\pi}>0, \tau_{q}>0, \mu>0$, $\kappa>0$, e $N=\nu \geq 0$. Kremer em [1], já havia mostrado a não negatividade da viscosidade volumétrica para gases ideais usando uma teoria termodinâmica estendida com 17 momentos. Aqui, em uma teoria de 14 momentos com uma formulação diferente, foi possível mostrar o mesmo para fluidos viscosos com condução de calor.

\section{Gases Ideais}

O caso em que a densidade de momento $\varrho_{i_{1} \ldots i_{n}}$ e o fluxo de momento $p_{i_{1} \ldots i_{n}}$ são idênticos representa uma teoria para gases ideais [4], [5]. Por isso, vale que $\left.\varrho_{s s}\right|_{E}=$ $\left.p_{s s}\right|_{E}=3 p_{0}$. 
Num gás ideal, já é conhecida a relação $\varepsilon=\frac{n}{2} \frac{p_{0}}{\varrho}$ onde $n$ representa o grau de liberdade molecular, que por sua vez, é igual a 3 para gases ideais monatômicos, 5 para diatômicos e 6 para poliatômicos. Mais ainda, para gases ideais, a equação de estado é dada por $p_{0}=R \varrho \theta$, onde $R=k / m$ é constante, $m$ é a massa atômica e $k$ é a constante de Boltzmann.

Os coeficientes citados em (6.2), (6.3) e (6.4) são explicitados abaixo:

$$
\begin{aligned}
\tau_{s} & =\frac{2}{r_{1}} R \varrho \theta^{2}, & \tau_{\pi} & =\frac{2}{r_{1}} \frac{n-3}{n} R \varrho \theta^{2}, & \tau_{q} & =\frac{10}{\tau} R^{2} \varrho \theta^{3}, \\
\mu & =\frac{2}{r_{1}} R^{2} \varrho^{2} \theta^{3}, & \kappa & =\frac{25}{\tau} R^{4} \varrho^{2} \theta^{4}, & \nu & =\frac{4}{3 r_{1}}\left(\frac{3-n}{n}\right)^{2} R^{2} \varrho^{2} \theta^{3}, \\
K & =\frac{2}{5} \frac{1}{R \varrho \theta}, & L & =-\frac{1}{R \varrho \theta}, & N & =\nu .
\end{aligned}
$$

Então $\nu=N=0$ somente para gases ideais monatômicos.

\section{Ondas Harmônicas}

Serão consideradas agora ondas harmônicas planas de amplitude pequena, a saber,

$$
\varrho=\tilde{\varrho}+\bar{\varrho} E, \quad v_{i}=\bar{v}_{i} E, \quad \theta=\tilde{\theta}+\bar{\theta} E, \quad S_{\langle i j\rangle}=\bar{S}_{\langle i j\rangle} E, \quad p_{d}=\bar{p}_{d} E, \quad q_{i}=\bar{q}_{i} E,
$$

onde $(\tilde{\varrho}, 0, \tilde{\theta}, 0,0,0)$ é um estado do gás em equilíbrio. Para um campo genérico $f, \bar{f}$ denota uma pequena amplitude complexa da propagação de uma pequena perturbação. Mais adiante $\tilde{f}$ denotará um campo $f$ avaliado no equilíbrio $(\varrho, \theta)=$ $(\tilde{\varrho}, \tilde{\theta})$. Nas relações acima, $E=e^{i(\omega t-k \boldsymbol{n} \cdot \boldsymbol{x})}$, onde $\omega$ é a freqüência, $k$ é o número de onda ( $k$ complexo), $\boldsymbol{n}$ a direção da propagação de onda ou normal da onda e $\boldsymbol{x}=\left(x_{1}, x_{2}, x_{3}\right) \in \mathbb{R}^{3}$. A velocidade de fase da onda $V_{p h}$ e a atenuação $\alpha$ são definidas por

$$
V_{p h}=\frac{\omega}{\operatorname{Re}(k)} \quad \text { e } \quad \alpha=-\operatorname{Im}(k)
$$

Admitimos (8.1) como solução para o sistema de equações formado pelas equações $(2.1)$ e (6.1) e desprezamos os termos de ordem dois, tais como os produtos entre $\bar{\varrho}, \bar{v}_{i}, \bar{\theta}, \bar{S}_{\langle i j\rangle}, \bar{p}_{d}, \bar{q}_{i}$. Consideramos o caso de propagação longitudinal na direção $\boldsymbol{n}=\boldsymbol{e}_{1}$ e com $\boldsymbol{v}=\left(v_{1}, 0,0\right)$. Neste caso particular, o sistema fica reduzido a um sistema linear $\mathbf{C X}=\mathbf{0}$. Para obter solução diferente da trivial, o determinante da matriz $\mathbf{C}$ deve ser nulo. Esta condição é conhecida como relação de dispersão, pela qual pode-se calcular a velocidade de fase $V_{p h}$ e a atenuação $\alpha$ em termos da freqüência $\omega$. O estudo foi restringido aos casos de freqüência alta e baixa em que é usado a fórmula de Taylor para aproximar $k / \omega$ por um polinômio.

\subsection{Gases ideais monatômicos}

Freqüência Alta: Para $\omega$ grande, as definições de velocidade de fase e atenuação 
(8.2) tornam-se

$$
\begin{aligned}
& \pm V_{p h}=\sqrt{\frac{15}{13 \pm \sqrt{94}}} \sqrt{\frac{\tilde{p}_{0}}{\tilde{\varrho}}}\left(1-\gamma_{2} \frac{1}{\omega^{2}}\right),
\end{aligned}
$$

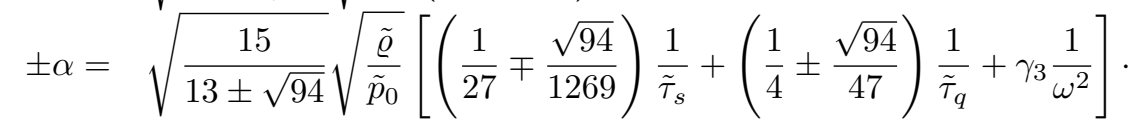

Em cada expressão acima, no menbro direito, as operações são realizadas todas com o sinal superior ou todas com o sinal inferior. O sinal \pm do membro esquerdo significa que há 4 valores para a velocidade de fase e 4 para a atenuação. Os coeficientes $\gamma_{2}$ e $\gamma_{3}$ são funções dependentes de $\tilde{\tau}_{s}$ e $\tilde{\tau}_{q}$.

Fazendo a freqüência $\omega$ tender para o infinito e admitindo a relação Maxwelliana $\tilde{\tau}_{s} / \tilde{\tau}_{q}=2 / 3$ (veja [6]), obtivemos os mesmos valores obtidos em [6] para velocidade de fase e atenuação de uma onda, para o caso monatômico.

Freqüência Baixa: Neste segundo caso, $\omega$ é próximo de zero e por (8.2), tem-se

$$
\begin{aligned}
\pm V_{p h} & =\sqrt{\frac{5}{3} \frac{\tilde{p}_{0}}{\tilde{\varrho}}}\left(1+\frac{1}{50}\left(16 \tilde{\tau}_{s}-\tilde{\tau}_{q}\right)\left(2 \tilde{\tau}_{s}+\tilde{\tau}_{q}\right) \omega^{2}\right), \\
\pm \alpha & =\frac{1}{5} \sqrt{\frac{3}{5} \frac{\tilde{\varrho}}{\tilde{p}_{0}}}\left(2 \tilde{\tau}_{s}+\tilde{\tau}_{q}\right) \omega^{2} .
\end{aligned}
$$

Liu e Müller já haviam mostrado em [5] resultados coerentes com os acima.

As outras possibilidades para velocidade de fase e atenuação são

$$
\begin{aligned}
\pm V_{p h} & =\sqrt{2 \tilde{\tau}_{q}} \sqrt{\frac{\tilde{p}_{0}}{\tilde{\varrho}}}\left(\omega^{1 / 2}-\frac{7}{10} \tilde{\tau}_{q} \omega^{3 / 2}\right), \\
\mp \alpha & =\sqrt{\frac{1}{2 \tilde{\tau}_{q}}} \sqrt{\frac{\tilde{\varrho}}{\tilde{p}_{0}}}\left(\omega^{1 / 2}-\frac{7}{10} \tilde{\tau}_{q} \omega^{3 / 2}\right) .
\end{aligned}
$$

Neste caso, $\lim _{\omega \rightarrow 0} V_{p h}=0, \lim _{\omega \rightarrow 0} \alpha=0$. Liu já havia mostrado em [5] que $\lim _{\omega \rightarrow 0} \alpha=0$.

\subsection{Gases ideais diatômicos, poliatômicos e outros}

Para simplificar, escreveremos abaixo $\gamma_{i}(n)$ em lugar de funções racionais em $n$ e dependentes de $\tilde{\tau}_{s}$ e $\tilde{\tau}_{q}$.

Freqüência Alta: Da definição de velocidade de fase e atenuação (8.2), tem-se

$$
\begin{aligned}
\pm V_{p h} & =\sqrt{\frac{15}{13 \pm \sqrt{94}}} \sqrt{\frac{\tilde{p}_{0}}{\tilde{\varrho}}}\left(1-\gamma_{2}(n) \frac{1}{\omega^{2}}\right) \\
\pm \alpha & =\sqrt{\frac{15}{13 \pm \sqrt{94}}} \sqrt{\frac{\varrho \varrho}{\tilde{p}_{0}}}\left(\frac{8413 \pm 451 \sqrt{94}}{42300} \frac{1}{\tilde{\tau}_{s}}+\frac{47 \pm 4 \sqrt{94}}{188} \frac{1}{\tilde{\tau}_{q}}+\gamma_{3}(n) \frac{1}{\omega^{2}}\right) .
\end{aligned}
$$

Ao fazer $\omega$ tender para o infinito, a velocidade de fase obtida neste caso coincide com a velocidade de fase obtida no caso monatômico (freqüência alta). 
Freqüência Baixa: Segundo (8.2),

$$
\begin{aligned}
\pm V_{p h} & =\sqrt{\frac{10 \tilde{\tau}_{q}}{n+2} \frac{\tilde{p}_{0}}{\tilde{\varrho}}}\left(-\omega^{1 / 2}-\gamma_{4}(n) \omega^{3 / 2}\right), \\
\mp \alpha & =\sqrt{\frac{n+2}{10 \tilde{\tau}_{q}} \frac{\tilde{\varrho}}{\tilde{p}_{0}}}\left(\omega^{1 / 2}+\gamma_{4}(n) \omega^{3 / 2}\right) .
\end{aligned}
$$

Ao fazer $n=3$, obtemos a velocidade de fase e a atenuação do caso monatômico.

As outras possibilidades para a velocidade de fase e atenuação são

$$
\pm V_{p h}=\sqrt{\frac{n+2}{n}} \sqrt{\frac{\tilde{p}_{0}}{\varrho}}\left(1+\gamma_{5}(n) \omega^{2}\right), \quad \pm \alpha=\sqrt{\frac{n}{n+2}} \sqrt{\frac{\tilde{\varrho}}{\tilde{p}_{0}}} \gamma_{6}(n) \omega^{2} .
$$

Ao fazer $n=3$, obtemos os mesmos valores que no caso monatômico a menos do coeficiente $\gamma_{5}(n)$. Era esperado obter $V_{p h}=\sqrt{\gamma} \frac{\tilde{\tilde{\tilde{\rho}}}}{\tilde{\varrho}}$ para $\omega \rightarrow 0$, com a razão entre o calor específico a pressão constante e o calor específico a volume constante $\gamma=c_{p} / c_{v}=5 / 3$ para gases ideais monatômicos, $\gamma=7 / 5$ para diatômicos, e $\gamma=4 / 3$ para poliatômicos, correspondentes com a termodinâmica clássica.

\section{Referências}

[1] G.M. Kremer, Extended thermodynamics of molecular ideal gases, Continuum Mech. Thermodyn., 1 (1989), 3-20.

[2] I-Shih Liu, Method of Lagrange multipliers for exploitation of the entropy principle, Arch. Rational Mech. Anal., 46 (1972), 131-148.

[3] I-Shih Liu, "Continuum Mechanics", Springer-Verlag Berlin Heidelberg New York (2002).

[4] I-Shih Liu, G.M. Kremer, Hyperbolic System of Field Equations for Viscous Fluids, Mat. Aplic. Comp., 9 (1990), 123-135.

[5] I-Shih Liu, I. Müller, Extended thermodynamics of classical and degenerate gases, Arch. Rational Mech. Anal., 83 (1983), 285-332.

[6] I. Müller, T. Ruggeri, "Rational Extended Thermodynamics", Second Edition, Springer-Verlag New York (1998). 